\title{
Limiting behavior of solutions of subelliptic heat equations
}

\author{
Federica DRAGONI \\ Scuola Normale Superiore \\ Piazza dei Cavalieri 7 \\ 56126 Pisa, Italy \\ e-mail: f.dragoni@sns.it
}

\begin{abstract}
We investigate the behavior, as $\varepsilon \rightarrow 0^{+}$, of $\varepsilon \log w^{\varepsilon}(t, x)$ where $w^{\varepsilon}$ are solutions of a suitable family of subelliptic heat equations. Using the Large Deviation Principle, we show that the limiting behavior is described by the metric inf-convolution w.r.t. the associated Carnot-Carathéodory distance.

2000 Mathematics Subject Classification: 35K70; 49J24; 53C17; 60F10.

Key words: Carnot-Carathéodory inf-convolutions; subelliptic heat equations; Large Deviation.
\end{abstract}

\section{Introduction}

It is well-known that the limiting behavior, as $\varepsilon \rightarrow 0^{+}$, of the solutions of

$$
\begin{cases}w_{t}^{\varepsilon}-\varepsilon \Delta w^{\varepsilon}=0, & x \in \mathbb{R}^{n}, t>0, \\ w^{\varepsilon}(0, x)=\mathrm{e}^{-\frac{g(x)}{2 \varepsilon}}, & x \in \mathbb{R}^{n} .\end{cases}
$$

is described by the Hamilton-Jacobi-Cauchy problem

$$
\left\{\begin{array}{cl}
u_{t}+\frac{1}{2}|D u|^{2}=0, & x \in \mathbb{R}^{n}, t>0, \\
u(0, x)=g(x), & x \in \mathbb{R}^{n},
\end{array}\right.
$$

see, for example, [20] and [4] or [2]. More precisely, if $g: \mathbb{R}^{n} \rightarrow \mathbb{R}$ is a bounded and continuous function, the logarithmic transform of $w^{\varepsilon}$, i.e. $u^{\varepsilon}=-2 \varepsilon \log w^{\varepsilon}$, converges, locally uniformly, as $\varepsilon \rightarrow 0^{+}$, to the unique viscosity solution $u$ of $(2)$. One way of proving this is to use both the representation of $w^{\varepsilon}$ as the integral 
convolution and the Hopf-Lax representation of the viscosity solution of (2) as the (euclidean) inf-convolution

$$
g_{t}(x)=\inf _{y \in \mathbb{R}^{n}}\left[g(y)+\frac{|x-y|^{2}}{2 t}\right],
$$

and to apply the Large Deviation Principle (see [18]) in order to establish the validity of the limiting relation

$$
\lim _{\varepsilon \rightarrow 0^{+}}-2 \varepsilon \log w^{\varepsilon}=u .
$$

The aim of this paper is to generalize the procedure described above in order to analyze the limiting behavior of some subelliptic diffusion equations in term of the Carnot-Carathéodory inf-convolutions. Let $w^{\varepsilon}$ the solutions of

$$
\left\{\begin{array}{c}
w_{t}^{\varepsilon}-\varepsilon \sum_{i, j=1}^{n} a_{i, j}(x) \frac{\partial^{2} w^{\varepsilon}}{\partial x_{i} \partial x_{j}}=0, \quad x \in \mathbb{R}^{n}, t>0, \\
w^{\varepsilon}(0, x)=\mathrm{e}^{-\frac{g(x)}{2 \varepsilon}}, \quad x \in \mathbb{R}^{n},
\end{array}\right.
$$

where the matrix $A(x)=\left(a_{i, j}(x)\right)_{i, j}$, for $i, j=1, \ldots, n$, is of the form

$$
A(x)=\sigma^{t}(x) \sigma(x),
$$

with $\sigma(x) m \times n$-matrix $(m \leq n)$, satisfying the Hörmander condition.

Under this condition, a finite Carnot-Carathéodory (C-C) distance can be associated by control theory to the matrix $\sigma$ (see [3]).

The inf-convolution of the initial datum $g$, with metric $d$ as kernel, namely

$$
g_{t}(x)=\inf _{y \in \mathbb{R}^{n}}\left[g(y)+\frac{d(x, y)^{2}}{2 t}\right],
$$

produces the viscosity solution of

$$
\left\{\begin{array}{cc}
u_{t}+\frac{1}{2}|\sigma(x) D u|^{2}=0, & x \in \mathbb{R}^{n}, t>0, \\
u(0, x)=g(x), & x \in \mathbb{R}^{n} .
\end{array}\right.
$$

Our main result is the following.

Theorem 1.1 Let $g \in C\left(\mathbb{R}^{n}\right)$ bounded, $d C$-C distance associated to an Hörmander's matrix $\sigma(x)$ and $g_{t}$ the inf-convolution defined by (4). If $w^{\varepsilon}$ are the solutions of (3), then

$$
\lim _{\varepsilon \rightarrow 0^{+}}-2 \varepsilon \log w^{\varepsilon}(t, x)=g_{t}(x),
$$

locally uniformly on $[0,+\infty) \times \mathbb{R}^{n}$.

Observe that in the special case $A(x)=I$, then $d(x, y)$ reduces to the standard euclidean distance, thus recovering the well-known classical result, see Capuzzo Dolcetta [4] for a recent presentation. 


\section{Preliminary results about Carnot- Carathéodory inf-convolutions and the Large Deviation Principle}

Let $d$ a distance on $\mathbb{R}^{n}$ and $g: \mathbb{R}^{n} \rightarrow \mathbb{R}$, then a metric inf-convolution can be defined, for any $t>0$, as (4). We look, in particular, at the C-C distances satisfying the Hörmander condition. We recall some notions about these, one can find more information in [3].

Fix $x \in \mathbb{R}^{n}$ and let $\sigma(x)$ a $m \times n$-matrix with $C^{\infty}$-coefficients and $m \leq n$. Setting $X_{1}(x), \ldots, X_{m}(x)$ the vector fields corresponding to the lines of $\sigma(x)$ (i.e. $\sigma(x)^{t}=$ $\left.\left[X_{1}(x), \ldots, X_{m}(t)\right]\right)$, we consider the control system

$$
\dot{\gamma}(t)=\sum_{i=1}^{m} \alpha_{i}(t) X_{i}(\gamma(t)),
$$

with $\alpha_{1}, \ldots, \alpha_{m}$ measurable real control functions.

We say that an absolutely continuous curve $\gamma:[0, T] \rightarrow \mathbb{R}^{n}$ is admissible or also $\sigma$-horizontal if there exists $\alpha:[0, T] \rightarrow \mathbb{R}^{m}$ measurable function such that

$$
\dot{\gamma}(t)=\sigma^{t}(\gamma(t)) \alpha(t), \quad \text { a.e. } t \in[0, T] .
$$

For any admissible curve $\gamma$ and any admissible coordinate-vector $\alpha(t)$, we define the length as

$$
l(\gamma)=\int_{0}^{T}\|\alpha(t)\| d t
$$

where $\|\quad\|$ is the standard euclidean norm in $\mathbb{R}^{m}$.

Remark 2.1 To get the uniqueness of the admissible coordinate-vector $\alpha(t)$, one can assume that the family of vector fields $X_{1}, \ldots, X_{m}$ satisfies the following weaklinear-independent condition: for all point $x$, there exist $1 \leq k \leq m$ and $1 \leq j_{1}<$ $\ldots<j_{k} \leq m$ such that

$$
\operatorname{rank}\left\{X_{j_{1}}(x), \ldots, X_{j_{k}}(x)\right\}=k, \text { and } X_{j}(x)=0, \quad \forall j \notin\left\{j_{1}, \ldots, j_{k}\right\} .
$$

If this condition doesn't hold, the length of an admissible curve can be defined as the infimum of the integrals (8) over all the admissible coordinates $\alpha(t)$.

Nevertheless a such condition is very general. In fact it holds for any generic distribution (so in particular for the Carnot groups) and for any Grušin-type space.

Definition 2.1 The C-C distance associated to $\sigma(x)$ is

$$
d(x, y)=\inf \{l(\gamma) \mid \gamma \sigma \text {-horizontal curve joining } x \text { to } y\} .
$$


In general, the function (9) is a distance on whole $\mathbb{R}^{n}$ but it is not always a finite distance. In order to overcome this problem, it is introduced the Hörmander condition. We recall that a bracket between two vector fields $X$ and $Y$ acts, by derivation, on all the smooth real functions $f$, as

$$
[X, Y] f=X(Y f)-Y(X f) .
$$

Let $\mathcal{L}^{0}=\left\{X_{1}, \ldots, X_{m}\right\}, \mathcal{L}^{1}=\left\{\left[X_{i}, X_{j}\right] \mid i, j=1, \ldots, m\right\}$ and

$$
\mathcal{L}^{k}=\left\{\left[Y_{i}, Y_{j}\right] \mid Y_{i} \in \mathcal{L}^{h}, Y_{j} \in \mathcal{L}^{l}, h, l=0, \ldots, k-1\right\} \backslash \bigcup_{i=0}^{k-1} \mathcal{L}^{i},
$$

then the Lie algebra associated to the distribution spanned by $X_{1}, \ldots, X_{m}$ is the set $\mathcal{L}=\bigcup_{k \in \mathbb{N}} \mathcal{L}^{k}$. We say that a matrix $\sigma(x)$ satisfies the Hörmander condition, if and only if, the associated Lie algebra spans whole of the tangent space, that in our case is $\mathbb{R}^{n}$, in any point.

If a matrix $\sigma(x)$ satisfies the Hörmander condition, the Chow's Theorem implies that the associated $\mathrm{C}-\mathrm{C}$ distance (9) is finite for any pair of points and it induces on $\mathbb{R}^{n}$ the euclidean topology (see $[3]$ ).

Moreover, we say that a matrix satisfies the Hörmander condition with step $k \geq 1$, if and only if,

$$
\bigcup_{i=1}^{k} \mathcal{L}^{i}(x)=\mathbb{R}^{n}
$$

in any point $x \in \mathbb{R}^{n}$. The Riemannian case is when $k=1$.

Now we give some examples of Hörmander's matrixes.

Example 2.1 The matrix

$$
\sigma(x)=\left(\begin{array}{ll}
1 & 0 \\
0 & x
\end{array}\right)
$$

satisfies the Hörmander condition with step 2 and it induces a sub-Riemannian geometry on $\mathbb{R}^{2}$, known as Grušin plane.

Example 2.2 Let

$$
\sigma(x)=\left(\begin{array}{ccc}
1 & 0 & -2 y \\
0 & 1 & 2 x
\end{array}\right)
$$

that is an Hörmander's matrix (with step 2) and it is associted to the 1-dimensional Heisenberg group.

Example 2.3 The following matrix satisfies the Hörmander condition with step 3 and the distribution associated to its lines is known as Martinet distribution:

$$
\sigma(x)=\left(\begin{array}{ccc}
1 & 0 & -y^{2} \\
0 & 1 & 0
\end{array}\right)
$$


For any C-C distance, with constant step $k \geq 1$, holds a locally euclidean estimate ([16]). In fact, for any $K \in \mathbb{R}^{n}$ compact, there exists a constant $C=C(K)>0$ such that

$$
\frac{1}{C}|x-y| \leq d(x, y) \leq C|x-y|^{\frac{1}{k}}, \quad \forall x, y \in K
$$

The metric inf-convolution (4) is a particular case of the Hopf-Lax function

$$
u(t, x)=\inf _{y \in \mathbb{R}^{n}}\left[g(y)+t \Phi^{*}\left(\frac{d(x, y)}{t}\right)\right],
$$

introduced by Manfredi-Stroffolini for the case of the Heisenberg group in [15] and generalized in $[4,5]$, see also [9]. We recall some properties proved in [9], rewritten directly for the inf-convolutions.

Theorem 2.1 Let $g \in L S C\left(\mathbb{R}^{n}\right)$ (lower semicontinuous) and bounded and $d C$ - $C$ distance, satisfying the Hörmander condition with step $k \geq 1$, then the metric inf-convolution $g_{t}$, defined in (4), is such that

(i) $g_{t} \leq g$, for any $t>0$,

(ii) the infimum in (4) is attended in the closed Carnot-Carathéodory ball, centered in $x$ and with radius $R(t)=2 t^{\frac{1}{2}}\|g\|_{\infty}^{\frac{1}{2}}$,

(iii) $g_{t}$ is locally d-Lipschitz in $x$ for $t>0$ and so, by estimate (10), is locally Hölder continuous with exponent $1 / k$. Moreover, $g_{t}$ is locally Lipschitz continuous in $t>0$, for any $x \in \mathbb{R}^{n}$,

(iv) $g_{t}$ monotonously converges (in the lower weak Barles-Perthame's sense, [2]) to $g$, as $t \rightarrow 0^{+}$,

(v) if $g(x) \leq-C(1+d(0, x))$, for some constant $C>0$, then

$$
g_{t}(x) \leq-C^{\prime}(1+t+d(0, x)),
$$

for any $x \in \mathbb{R}^{n}$ and $t>0$, with $C^{\prime}=\max \left\{C, \frac{1}{2} C^{2}\right\}$.

So the Carnot-Carathéodory inf-convolutions (4) are a monotonous Lipschitz approximation of the original function as in the euclidean case (see [1]). Moreover in [9] (Theorem 4.1) we have proved that $u(t, x)=g_{t}(x)$ solves (in the viscosity sense) the Cauchy problem (5). At last, when $g$ is continuous, the solution of (5) is also unique (see [6]).

We recall that, if $\sigma(x)$ is an Hörmander's matrix, then the differential operator $L:=\sum_{i, j} a_{i, j}(x) \partial_{x_{i}} \partial_{x_{j}}$ is hypoelliptic.

By theory of subelliptic heat equations (see [10, 12]), we know that there exists an heat kernel associated to $L$, which we indicate by $p(t, x, y)$, smooth, for $t>0$, 
in whole $\mathbb{R}^{n} \times \mathbb{R}^{n}$ and, moreover, there exists $M \in[1,+\infty)$ such that, for any $0<t \leq 1$ and $x, y \in \mathbb{R}^{n}$, it holds

$$
\frac{1}{M \mu\left(B^{d}(x, \sqrt{t})\right)} \mathrm{e}^{-M \frac{d(x, y)^{2}}{t}} \leq p(t, x, y) \leq \frac{M}{\mu\left(B^{d}(x, \sqrt{t})\right)} \mathrm{e}^{-\frac{d(x, y)^{2}}{M t}} .
$$

see [11]. At last, let $p^{\varepsilon}(t, x, y)$ the heat kernel associated to $\varepsilon L$, the solution of (3) is given by

$$
w^{\varepsilon}(t, x)=\int_{\mathbb{R}^{n}} \mathrm{e}^{-\frac{g(x)}{2 \varepsilon}}(y) p^{\varepsilon}(t, x, y) d y .
$$

So, in order to prove Theorem 1.1, we need to investigate the limiting behavior of

$$
u^{\varepsilon}(t, x)=-2 \varepsilon \log \left(\int_{\mathbb{R}^{n}} e^{-\frac{g(y)}{2 \varepsilon}} p^{\varepsilon}(t, x, y) d y\right) .
$$

As in [4], we want to apply a Laplace-Varadhan's type theorem, that is an application of the Large Deviation Principle.

Now we recall both of these results, for some information about the Large Deviation theory, one can see $[18]$ or also $[7,8]$.

Definition 2.2 (Large Deviation Principle). Let $P_{\varepsilon}$ a family of probability measures, defined on the borel sets of a complete and separable metric space $X$. A family $P_{\varepsilon}$ satisfies the Large Deviation Principle (LDP) if there exists a function (called rate function) $I: X \rightarrow[0,+\infty]$ such that

(i) $I \in L S C(X)$,

(ii) for any $k<+\infty$, the sublevel set $\{y \in X \mid I(y) \leq k\}$ is compact,

(iii) for any $A \subset X$ open set,

$$
\liminf _{\varepsilon \rightarrow 0^{+}} \varepsilon \log P_{\varepsilon}(A) \geq-\inf _{y \in A} I(y),
$$

(iv) for any $C \subset X$ closed set,

$$
\limsup _{\varepsilon \rightarrow 0^{+}} \varepsilon \log P_{\varepsilon}(C) \leq-\inf _{y \in C} I(y) .
$$

Theorem 2.2 Let $X$ a complete and separable metric space and $P_{\varepsilon}$ a family of probability measures satisfying the LDP with rate function I, then, for any $F \in C(X)$ bounded,

$$
\lim _{\varepsilon \rightarrow 0^{+}} \varepsilon \log \left(\int_{X} \exp \left[\frac{F(y)}{\varepsilon}\right] d P_{\varepsilon}(y)\right)=\sup _{y \in X}[F(y)-I(y)] .
$$


Let $X=\mathbb{R}^{n}$ and fixed $x \in \mathbb{R}^{n}$ and $t>0$. We can define, for any $B \subset \mathbb{R}^{n}$ borel set, the following probability measures

$$
P_{\varepsilon}^{t, x}(B)=\int_{B} p^{\varepsilon}(t, x, y) d y
$$

If we show that previous family of probability measures (15) satisfies the LDP with rate function

$$
I^{t, x}(y)=\frac{d(x, y)^{2}}{4 t}
$$

by Theorem 2.2 with $F=-g / 2$, it is immediate to get (6). The difficulty is to verify properties (iii) and (iv). If $p^{\varepsilon}$ is the heat kernel associated to some uniformly elliptic operators there is a non-trivial proof of this fact in [20] (note that, in a such case, $d$ is a Riemannian distance). Nevertheless, in the euclidean case, it is enough easy to get properties (iii) and ( $i v)$ (as unique limit) in the borel and bounded sets. In fact, setting $q=1 / \varepsilon$, by the convergence of the $L^{q}$-norm to the $L^{\infty}$-norm, as $q \rightarrow+\infty$, one can deduce directly that

$$
\lim _{\varepsilon \rightarrow 0^{+}} \varepsilon \log \left((4 \pi \varepsilon t)^{-\frac{n}{2}} \int_{B} \mathrm{e}^{-\frac{|x-y|^{2}}{4 \varepsilon t}} d y\right)=\log \left(\lim _{q \rightarrow+\infty}\left\|\mathrm{e}^{-\frac{|x-\cdot|}{4 t}}\right\|_{q, B}\right)=-\inf _{y \in B} \frac{|x-y|^{2}}{4 t} .
$$

This remark has given us the idea for an analytic proof which covers also the Carnot-Carathéodory case.

\section{Proof of the main result}

To apply LDP in order to get Theorem 1.1, we need to generalize to the hypoelliptic case the asymptotic estimates, proved in [20] for uniform elliptic operators. Next lemma is a key-point.

Lemma 3.1 Let $p(t, x, y)$ the heat kernel associated to $A$, then

$$
p^{\varepsilon}(t, x, y)=p(\varepsilon t, x, y) .
$$

Proof. The result follows immediately from the uniqueness for the Cauchy problem:

$$
\left\{\begin{array}{c}
\frac{\partial}{\partial t} p^{\varepsilon}(t, x, y)-\varepsilon \sum_{i, j} a_{i, j}(x) \frac{\partial^{2}}{\partial x_{i} \partial x_{j}} p^{\varepsilon}(t, x, y)=0, \quad x, y \in \mathbb{R}^{n}, t>0 \\
p^{\varepsilon}(0, x, y)=\delta_{x}(y), \quad x, y \in \mathbb{R}^{n} .
\end{array}\right.
$$

In fact, since the coefficients of the equation don't depend on the time-variable, it is trivial that $p(\varepsilon t, x, y)$ satisfies the Cauchy problem (17).

So, by the uniqueness (see [12]), we can conclude. 
The second key-result is the following locally uniform limit, proved in [19] for uniformly elliptic operators and generalized to the hypoelliptic case in [14, 13].

Theorem 3.1 Let $p(t, x, y)$ as in Lemma 3.1 and $d(x, y)$ the $C$ - $C$ distance defined in (9), then

$$
\lim _{\tau \rightarrow 0^{+}} 4 \tau \log p(\tau, x, y)=-d(x, y)^{2}
$$

Moreover previous convergence is uniform in the bounded sets.

The idea is to use previous results in order to investigate the limiting behavior of $\left(P_{\varepsilon}^{t, x}\right)^{\varepsilon}$ in the bounded sets and then deduce the behavior in the open and closed (unbounded) sets.

Proposition 3.1 Let $p^{\varepsilon}(t, x, y)$ the heat kernel associated to the hypoelliptic operator $L^{\varepsilon}=\varepsilon \sum_{i, j} a_{i, j}(x) \frac{\partial^{2}}{\partial x_{j} \partial x_{i}}$, with $A(x)=\left(a_{i, j}(x)\right)_{i, j=1}^{n}=\sigma^{t}(x) \sigma(x)$ and $\sigma(x)$ Hörmander's matrix. If $P_{\varepsilon}^{t, x}$ and $I^{t, x}$ are the family of probability measures and the rate function defined in (15) and (16), respectively, fix $t>0, x \in \mathbb{R}^{n}$, then, for any $B \subset \mathbb{R}^{n}$ bounded set,

$$
\lim _{\varepsilon \rightarrow 0^{+}}\left[P_{\varepsilon}^{t, x}(B)\right]^{\varepsilon}=\mathrm{e}^{-\inf _{y \in B} I^{t, x}(y)} .
$$

Proof. We use the exponential form of the uniform limit (18), that is

$$
\lim _{\tau \rightarrow 0^{+}} p(\tau, x, y)^{\tau}=\mathrm{e}^{-\frac{d(x, y)^{2}}{4}}
$$

Let $q=\frac{1}{\tau}$ and $f_{q}(y)=p\left(\frac{1}{q}, x, y\right)^{\frac{1}{q}}$, we can write

$$
\left(\int_{B}\left[p(\tau, x, y)^{\tau}\right]^{\frac{1}{\tau}} d y\right)^{\tau}=\left(\int_{B}\left[p\left(\frac{1}{q}, x, y\right)^{\frac{1}{q}}\right]^{q} d y\right)^{\frac{1}{q}}=\left\|f_{q}\right\|_{q, B}
$$

where by \|\|$_{q, B}$ we indicate the usual $L^{q}$-norm in $B$, with $q \geq 1$.

By Lemma 3.1 and setting $\tau=\varepsilon t$, we get

$$
\lim _{\varepsilon \rightarrow 0^{+}}\left[P_{t, x}^{\varepsilon}(B)\right]^{\varepsilon}=\lim _{\tau \rightarrow 0^{+}}\left(\int_{B} p(\tau, x, y) d y\right)^{\frac{\tau}{t}}=\lim _{q \rightarrow+\infty}\left\|f_{q}\right\|_{q, B}^{\frac{1}{t}} .
$$

Set $f(y)=\mathrm{e}^{-\frac{d(x, y)^{2}}{4}}$, by the triangle inequality for the $L^{q}$-norm, we get

$$
\left|\left\|f_{q}\right\|_{q, B}-\|f\|_{q, B}\right| \leq\left\|f_{q}-f\right\|_{q, B} \leq\left\|f_{q}-f\right\|_{\infty, B}[\mu(B)]^{\frac{1}{q}} .
$$


As $q \rightarrow \infty$, the last member goes to zero and $\|f(y)\|_{q, B} \rightarrow\|f(y)\|_{\infty, B}$, since $B$ is bounded. So we get

$$
\lim _{q \rightarrow+\infty}\left\|f_{q}\right\|_{q, B}^{\frac{1}{t}}=\sup _{y \in B}|f(y)|^{\frac{1}{t}}=\mathrm{e}^{-\inf _{y \in B} \frac{d(x, y)^{2}}{4 t}} .
$$

Hence, by (20), the convergence result (19) holds.

To get, by approximation, the corresponding estimate for the lower-limit in the open (unbounded) sets, is very easy.

Proposition 3.2 Under assumptions of Proposition 3.1, then, for any open set $A \subset \mathbb{R}^{n}$

$$
\liminf _{\varepsilon \rightarrow 0^{+}}\left[P_{\varepsilon}^{t, x}(A)\right]^{\varepsilon} \geq \mathrm{e}^{-\inf _{y \in A} I^{t, x}(y)} .
$$

Proof. Let $A_{R}:=A \cap B_{R}(0)$. Since $A_{R}$ is bounded, we can apply the limiting behavior proved in Proposition 3.1 and so

$$
\liminf _{\varepsilon \rightarrow 0^{+}}\left[P_{\varepsilon}^{t, x}(A)\right]^{\varepsilon} \geq \liminf _{\varepsilon \rightarrow 0^{+}}\left[P_{\varepsilon}^{t, x}\left(A_{R}\right)\right]^{\varepsilon}=\mathrm{e}^{-\inf _{y \in A_{R}} \frac{d(x, y)^{2}}{4 t}} .
$$

Taking the supremum for $R>0$, we can immediately conclude that

$$
\liminf _{\varepsilon \rightarrow 0^{+}}\left[P_{\varepsilon}^{t, x}(A)\right]^{\varepsilon} \geq \sup _{R>0} \mathrm{e}^{-\inf _{y \in A_{R}} \frac{d(x, y)^{2}}{4 t}} \geq \mathrm{e}^{-\inf _{y \in A} \frac{d(x, y)^{2}}{4 t}} .
$$

To get the estimate for the upper-limit in the closed (unbounded) sets, is more complicate and, first, we need to investigate the limiting behavior outside large balls.

Lemma 3.2 Let $\delta \in(0,1)$, then there exists $R_{\delta}>0$ such that

$$
\limsup _{\tau \rightarrow 0^{+}}\left(\int_{\mathbb{R}^{n} \backslash \frac{B_{R_{\delta}}^{d}(x)}{}} p(\tau, x, y) d y\right)^{\tau}<\delta .
$$

Proof. Set $B_{R}^{-}=\mathbb{R}^{n} \backslash \bar{B}_{R}^{d}(x)$, by the Hörmander assumption, it is well-known (see [17]) that there exists $c>0$ such that

$$
B\left(x, c \tau^{\frac{k}{2}}\right) \subset B^{d}(x, \sqrt{\tau}),
$$

where $k \geq 1$ is the step of the distribution associated to $X_{1}, \ldots, X_{m}$, then $\mu\left(B^{d}(x, \sqrt{\tau})\right)^{-1} \leq\left(\omega_{n} c^{n} \tau^{\frac{n k}{2}}\right)^{-1}$, with $\omega_{n}$ measure of the unit euclidean ball. By estimate (11) and setting $\lambda=M \omega_{n}^{-1} c^{-n}>0$, we get

$$
\limsup _{\tau \rightarrow 0^{+}}\left(\int_{B_{R}^{-}} p(\tau, x, y) d y\right)^{\tau} \leq \limsup _{\tau \rightarrow 0^{+}} \lambda^{\tau} \tau^{-\frac{n k}{2} \tau}\left(\int_{B_{R}^{-}} \mathrm{e}^{-\frac{d(x, y)^{2}}{M \tau}} d y\right)^{\tau} .
$$


It is trivial that $\lim _{\tau \rightarrow 0^{+}}\left(\lambda \tau^{-\frac{n k}{2}}\right)^{\tau}=1$, so it remains to estimate

$$
L_{R}=\limsup _{\tau \rightarrow 0^{+}}\left(\int_{B_{R}^{-}} \mathrm{e}^{-\frac{d(x, y)^{2}}{M \tau}} d y\right)^{\tau} .
$$

Using the continuity of the logarithm function, we study $\log L_{R}$ and apply a version of the De l'Hôpital Theorem for the upper-limit. In fact, by the Cauchy Theorem, it is easy to show that

$$
\limsup _{\tau \rightarrow 0^{+}} \frac{f(\tau)}{g(\tau)} \leq \limsup _{\tau \rightarrow 0^{+}} \frac{f^{\prime}(\tau)}{g^{\prime}(\tau)}
$$

whenever $f$ and $g$ are continuous differentiable. Then

$$
\log L_{R}=\limsup _{\tau \rightarrow 0^{+}} \frac{\log \left(\int_{B_{R}^{-}} \mathrm{e}^{-\frac{d(x, y)^{2}}{M \tau}} d y\right)}{\frac{1}{\tau}} \leq \limsup _{\tau \rightarrow 0^{+}}-\tau^{2} \frac{\int_{B_{R}^{-}} \mathrm{e}^{-\frac{d(x, y)^{2}}{M \tau}} \frac{d^{2}(x, y)}{M \tau^{2}} d y}{\int_{B_{R}^{-}} \mathrm{e}^{-\frac{d(x, y)^{2}}{M \tau}} d y}
$$

Since $y \in \mathbb{R}^{n} \backslash \bar{B}_{R}^{d}(x)$, then $d(x, y) \geq R$. Therefore we get

$$
\log L_{R} \leq \limsup _{\tau \rightarrow 0^{+}}-\frac{R^{2}}{M} \frac{\int_{B_{R}^{-}} \mathrm{e}^{-\frac{d(x, y)^{2}}{M \tau}} d y}{\int_{B_{R}^{-}} \mathrm{e}^{-\frac{d(x, y)^{2}}{M \tau}} d y}=-\frac{R^{2}}{M} .
$$

We can conclude that, for any $R>0$,

$$
\limsup _{\tau \rightarrow 0^{+}}\left(\int_{B_{R}^{-}} p(\tau, x, y) d y\right)^{\tau} \leq \mathrm{e}^{-\frac{R^{2}}{M}}
$$

Hence, for any $0<\delta<1$, we can choose $R_{\delta}>\sqrt{\frac{-\log \delta}{M}}$ so that $\mathrm{e}^{-\frac{R_{\delta}^{2}}{M}}<\delta$ and this concludes the proof.

Proposition 3.3 Under assumptions of Proposition 3.1, then, for any closed set $C \subset \mathbb{R}^{n}$,

$$
\limsup _{\varepsilon \rightarrow 0^{+}}\left[P_{\varepsilon}^{t, x}(C)\right]^{\varepsilon} \leq \mathrm{e}^{-\inf _{y \in C} I^{t, x}(y)} .
$$

Proof. As for the bounded sets, let $\tau=\varepsilon t$, instead of (22), we can show

$$
\limsup _{\tau \rightarrow 0^{+}}\left(\int_{C} p(\tau, x, y) d y\right)^{\tau} \leq e^{-\inf _{y \in C} \frac{d(x, y)^{2}}{4}}
$$


Since $\tau \in(0,1)$, for any $\delta \in(0,1)$, we can decompose $C=C_{\delta} \cup C_{\delta}^{-}$, where $C_{\delta}=C \cap \bar{B}_{R_{\delta}}^{d}(x)$ and $C_{\delta}^{-}=C \backslash \bar{B}_{R_{\delta}}^{d}(x)$. In the bounded set $C_{\delta}$ we can apply Proposition 3.1 while in $C_{\delta}^{-}$we can use Lemma 3.2, so

$$
\begin{aligned}
& \limsup _{\tau \rightarrow 0^{+}}\left(\int_{C} p(\tau, x, y) d y\right)^{\tau} \leq \lim _{\tau \rightarrow 0^{+}}\left(\int_{C_{\delta}} p(\tau, x, y) d y\right)^{\tau}+ \\
& \limsup _{\tau \rightarrow 0^{+}}\left(\int_{C_{\delta}^{-}} p(\tau, x, y) d y\right)^{\tau} \leq e^{-\inf _{y \in C_{\delta}} \frac{d(x, y)^{2}}{4}}+\delta \leq e^{-\inf _{y \in C} \frac{d(x, y)^{2}}{4}}+\delta .
\end{aligned}
$$

Passing to the limit as $\delta \rightarrow 0^{+}$, we get estimate (23).

Finally we can give the proof of the main result.

Proof of Theorem 1.1 We remark that, since $d$ is a C-C distance, properties $(i)$ and $(i i)$ of the Large Deviation Principle hold. In fact, we have already remarked that the Hörmander condition implies that $d$ induces on $\mathbb{R}^{n}$ the euclidean topology. It means that $d$ is continuous and the sublevels are compact sets.

Moreover, since the logarithm is a continuous and non decreasing function, Propositions 3.2 and 3.3 give properties $(i i i)$ and $(i v)$ of the Large Deviation Principle. Applying the Large Deviation Theorem 2.2 with $F(y)=\mathrm{e}^{-\frac{g}{2}(y)}$ we find the convergence result (6).

Remark 3.1 Note that this gives also an alternative proof for the result showed in $[20]$.

Moreover, we want to remark that

$$
\left\{\begin{array}{cc}
u_{t}^{\varepsilon}-\varepsilon \sum_{i, j=1}^{n} a_{i, j}(x) \frac{\partial^{2} u^{\varepsilon}}{\partial x_{i} \partial x_{j}}+\frac{1}{2}\left|\sigma(x) D u^{\varepsilon}\right|^{2}=0, \quad x \in \mathbb{R}^{n}, t>0, \\
u^{\varepsilon}(0, x)=g(x), \quad x \in \mathbb{R}^{n},
\end{array}\right.
$$

gives a second-order approximation of the Cauchy problem (5).

By the Hopf-Cole transform $w^{\varepsilon}=\mathrm{e}^{-\frac{u^{\varepsilon}}{2 \varepsilon}}$, we can linearize problem (24). In fact, setting $A(x)=\sigma^{t}(x) \sigma(x)$, we find

$$
w_{t}^{\varepsilon}-\varepsilon \sum_{i, j=1}^{n} a_{i, j}(x) \frac{\partial^{2} w^{\varepsilon}}{\partial x_{i} \partial x_{j}}=-\frac{w^{\varepsilon}}{2 \varepsilon}\left(u_{t}^{\varepsilon}-\varepsilon \sum_{i, j=1}^{n} a_{i, j}(x) \frac{\partial^{2} u^{\varepsilon}}{\partial x_{i} \partial x_{j}}-\frac{1}{2} A(x) D u^{\varepsilon} \cdot D u^{\varepsilon}\right) .
$$

Remarking that

$$
\left|\sigma(x) D u^{\varepsilon}\right|^{2}=\sigma(x) D u^{\varepsilon} \cdot \sigma(x) D u^{\varepsilon}=\sigma^{t}(x) \sigma(x) D u^{\varepsilon} \cdot D u^{\varepsilon}=A(x) D u^{\varepsilon} \cdot D u^{\varepsilon},
$$

we get that, if $u^{\varepsilon}$ solves the Cauchy problem (24), then its Hopf-Cole transform $w^{\varepsilon}$ solves exactly the Cauchy problem (3). 
So it is natural that Theorem 1.1 holds, because it means the convergence of the solutions of the approximating problem (24), i.e. $u^{\varepsilon}=-2 \varepsilon \log w^{\varepsilon}$, to the unique viscosity solution of the original Cauchy problem.

\section{Acknowledgements}

I want to thank Prof. I. Capuzzo Dolcetta; without his help this work would not have came to be.

Moreover I want to thank also Prof. L. Ambrosio and Prof. R. Magnanini for the useful suggestions.

\section{References}

[1] Ma. BARDI and I. CAPUZZO DOLCETTA, Optimal Control and Viscosity Solutions of Hamilton-Jacobi-Bellman Equations, Birkhäuser, Boston, 1997.

[2] G. BARLES, Discontinuous viscosity solutions of first-order Hamilton-Jacobi equations: a guided visit, Nonlinear Anal. 20(9) (1993), 1123-1134.

[3] A. BELLAÏCHE, Sub-Riemannian Geometry, Basel, Birkhauser, 1996.

[4] I. CAPUZZO DOLCETTA, Representations of solutions of Hamilton-Jacobi equations, Progr. Nonlinear Diff. Eq. Appl. 54, Birkhäuser, Basel, 2003.

[5] I. CAPUZZO DOLCETTA and H. ISHII, Hopf formulas for state-dependent Hamilton-Jacobi equation, preprint.

[6] A. CUTRÌ and F. DA LIO, Comparison and uniqueness results for noncoercitive first-order Hamilton-Jacobi equations, to appear on ESAIM-COV.

[7] A. DEMBO and O. ZEITOUNI, Large Deviation and applications, SpringerVerlag, New York, 1998.

[8] F. DEN HOLLANDER, Large Deviations, Am. Math. Soc., Providence, 2000.

[9] F. DRAGONI, Metric Hopf-Lax formula with semicontinuous data, preprint.

[10] D. S. JERISON and A. SÁNCHEZ-CALLE, Estimates for the heat kernel for a sum of squares of vector fields, Ind. Univ. Math. J. 35(4) (1986), 835-854.

[11] S. KUSUOKA and D. STROOCK, Applications of Malliavin calculus, part III, J. Fac. Sci. Univ. Tokyo Sect. 1A Math. 34(2) (1987), 391-442.

[12] E. LANCONELLI and A. PASCUCCI, On the fundamental solution for hypoelliptic second order partial differential equations with non-negative characteristic form, Ricerche Mat. 48(1) (1999), 81-106. 
[13] R. LÉANDRE, Majoration en temps petit de la densité d'une diffusion dégénérée, Prob. Theory Related Fields 74(2) (1987), 289-294.

[14] R. LÉANDRE, Minoration en temps petit de la densité d'une diffusion dégénérée, Journal of Functional Analysis 74(2) (1987), 399-414.

[15] J. J. MANFREDI and B. STROFFOLINI, A version of the Hopf-Lax formula in the Heisenberg group, Comm. in Partial Diff. Equations 27(5-6) (2002), 1139-1159.

[16] A. NAGEL, E. M. STEIN and S. WAINGER, Balls and metrics defined by vector fields I: basic properties, Acta Math. 155 (1985), 103-147.

[17] A. SÁNCHEZ-CALLE, Fundamental solutions and geometry of the sum of squares of vector fields, Invent. Math. 78 (1984), 143-160.

[18] S. R. S. VARADHAN, Large Deviations and Applications, Society for Industrial and Applied Mathematics, Philadelphia PA, 1984

[19] S. R. VARADHAN, On the behavior of the fundamental solution of the heat equation with variable coefficients, Coom. Pure Appl. Math. 20 (1967), $431-455$.

[20] S. R. VARADHAN, Diffusion Processes in a Small Time Interval, Coom. Pure Appl. Math. 20 (1967), 659-685.

Received 20 March 2006; accepted 7 July 2006

To access this journal online: http://www.birkhauser.ch 